\title{
Cell adhesion in renal tubular epithelial cells: Biochemistry, biophysics or both
}

\author{
Claire Elizabeth Hills; Eleftherios SIAMANTOURAS; Paul Edward SQUIRES* \\ Diabetes, Metabolism \& Inflammation Group, School of Life Sciences, Joseph Banks Laboratories, University of Lincoln, Lincoln, LN6 7DL, UK
}

Key words: Cell-adhesion, Cadherin, Cytoskeleton, AFM, Kidney, Epithelium

\begin{abstract}
Changes in cell-cell and cell-substrate adhesion markers are increasingly used to characterize disease onset and progression. However, these relationships depend on both the biochemical and molecular association between cells and between cells and their extracellular matrix, as well as the biophysical and mechanical properties orchestrated by cytoskeletal, membrane and matrix components. To fully appreciate the role of cell adhesion when determining normal physiology and the impact of disease on cellular function, it is important to consider both biochemical and biophysical attributes of the system being investigated. In this short viewpoint we reflect on our experiences assessing cell-cell and/or cell-matrix interactions in renal tubular epithelial cells.
\end{abstract}

\section{Introduction}

Cell-cell and cell-substrate interactions are dynamic and complex and rely on both biochemical association between cell adhesion molecules and biophysical properties of the cell membrane and cytoskeleton. Arranged at the point of cell-cell or cell-matrix contact, adhesion protein complexes directly connect with the actin cytoskeleton and relay information from the local microenvironment or neighboring cells to affect intracellular structure and function. Changes in cell adhesion often represent defining events in a range of diseases, including cancer. However, understanding how changes in cell adhesion impact on disease onset and progression depends on an understanding for both biochemical homotypic and heterotypic cell-cell interactions and cell-substrate contacts, as well as an appreciation for biophysical determinants such as structural organization of the cytoskeleton and/or extracellular matrix. This brief viewpoint focusses on our use of renal tubular epithelial cells and the need to consider both biochemical and biophysical aspects of cell-cell and cell-substrate adhesion when determining effects of disease on cellular function.

\section{Cell-to-Cell Interactions}

Physical coupling between cells and between cells and their immediate environment is a multifaceted process involved

*Address correspondence to: Paul Edward Squires,

PSquires@lincoln.ac.uk

Received: 23 July 2021; Accepted: 23 August 2021 in differentiation, proliferation and cell migration and is fundamental to the development and maintenance of tissue function. In tubular epithelia of the diabetic kidney, loss of the adhesion protein epithelial (E)-cadherin has been linked to early structural and functional changes associated with disease onset and progression (Hills et al., 2012). The extracellular domain of E-cadherin form weak ligations with similar proteins on adjacent cells, whilst the cytoplasmic domain binds beta-catenin, linking E-cadherin to cytoskeletal filamentous (F)-actin via alpha-catenin. The cadherin-catenin complex is force-sensitive (Buckley et al., 2014), and interaction with the actin cytoskeleton increases adhesive strength of the junction and acts as a signaling node for proteins that influence cell tethering and/or initiate intracellular signaling. In disease, e.g., diabetic nephropathy and/or tubulointerstitial fibrosis, reduced E-cadherin expression and a decrease in the number of associated ligations, lessens the adhesive strength between cells and contributes to a loss of cell-cell contact (Siamantouras et al., 2016). This has implications for increased paracellular permeability and decreased gap-junction intercellular communication (Hills et al., 2018). It has been suggested that gap-junctions themselves exhibit adhesive characteristics (Cotrina et al., 2008; Lin et al., 2002) and the contribution of connexin (Cx)-mediated gap junctions to cell-cell stickiness and barrier function has recently been considered (Strauss and Gourdie, 2020).

There is no doubt that adhesion molecules have a predictive, diagnostic and therapeutic role (Wennstrom and Nielsen, 2012; Jaitovich and Jaim Etcheverry, 2004). However, the expression/function of adhesion proteins is 
not the sole determinant of the energy required to uncouple cells and should not be used in isolation to identify loss of cell-cell contact without determining functional parameters associated with detachment. In addition to the number of molecular ligations, cell-cell tethering is related to elastic resistance of the cell in response to mechanical deformation during cell-cell separation (Siamantouras et al., 2015; Siamantouras et al., 2019). Cellular elasticity is a biophysical property largely associated with 3-dimensional cytoskeletal architecture. Made up of a network of filamentous proteins, the cytoskeleton provides an adaptable physical scaffold that facilitates various cell and tissue functions. In disease, this complex interconnected skeleton can be reorganized into rigid peripheral stress fibers (reviewed in Kassianidou and Kumar (2015)). Orientation of cytoskeletal elements into bundles of actin around the cell periphery, exhibits different tensile strength and elastic properties (distance of separation) compared to the diffuse transcellular network more commonly associated with healthy conditions (as shown in Fig. 1). Deformation characteristics at the cellular level can provide important information about cell stiffness, migratory capacity and the development and advancement of disease, e.g., partial epithelial-to-mesenchymal transition in diabetic nephropathy (Hills and Squires, 2011), or tumor development and metastasis (Scarpa et al., 2015), and should be considered alongside biochemical changes to adhesion proteins.

\section{Cell-to-Substrate Interactions}

As bidirectional hubs for the transfer of information between cell and substrate, integrins and integrin-mediated adhesions connect cells to their extracellular matrix (ECM) and facilitate efficient and appropriate tissue-specific function in response to local external stimuli (Kechagia et al., 2019). Imbalance between substrate synthesis and degradation contributes to renal fibrosis, and changes in the amount or composition of matrix proteins can alter membrane receptor-ECM attachment, modify signal transduction and ultimately switch cell phenotype (Theocharis et al., 2019). Cell-cell and cell-ECM interactions are reciprocally linked, and a loss of cell-cell adhesion, can change cell-ECM tethering, alter deformation characteristics and adjust the way cells interact with one another (Goodwin et al., 2017). We recently demonstrated that up-regulation of the ECM protein collagen I exacerbates damage and modifies phenotype in renal tubular epithelial cells via a2 $\beta 1 /$ integrinlinked kinase-1 regulated connexin-mediated activity (Potter et al., 2021). With evidence that injured tubule cells preincubated with connexin-43 (Cx43) hemichannel blocker Peptide 5 (Peptagon $^{\mathrm{TM}}$ ) exhibit decreased collagen I secretion, these data suggest that blocking Cx43 mediated activity at the cell-cell interface may negate a feedback loop, in which the ECM substrate collagen I exacerbates Cx43 hemichannel activity, which further drives increased secretion of collagen I and impacts on phenotypic change (Price et al., 2020).

Cell-ECM interconnections are subject to rapid change either physiologically e.g., during development, or in disease e.g., renal inflammation and fibrosis. In cancer, stiffening of the ECM can enhance substrate tethering through increases in adhesion protein expression and cytoskeletal tension (reviewed in Gkretsi and Stylianopoulos (2018)). With ECM remodelling known to promote a loss of epithelial stability, it is important to consider both the biochemical and biophysical relationship between cell and substrate when
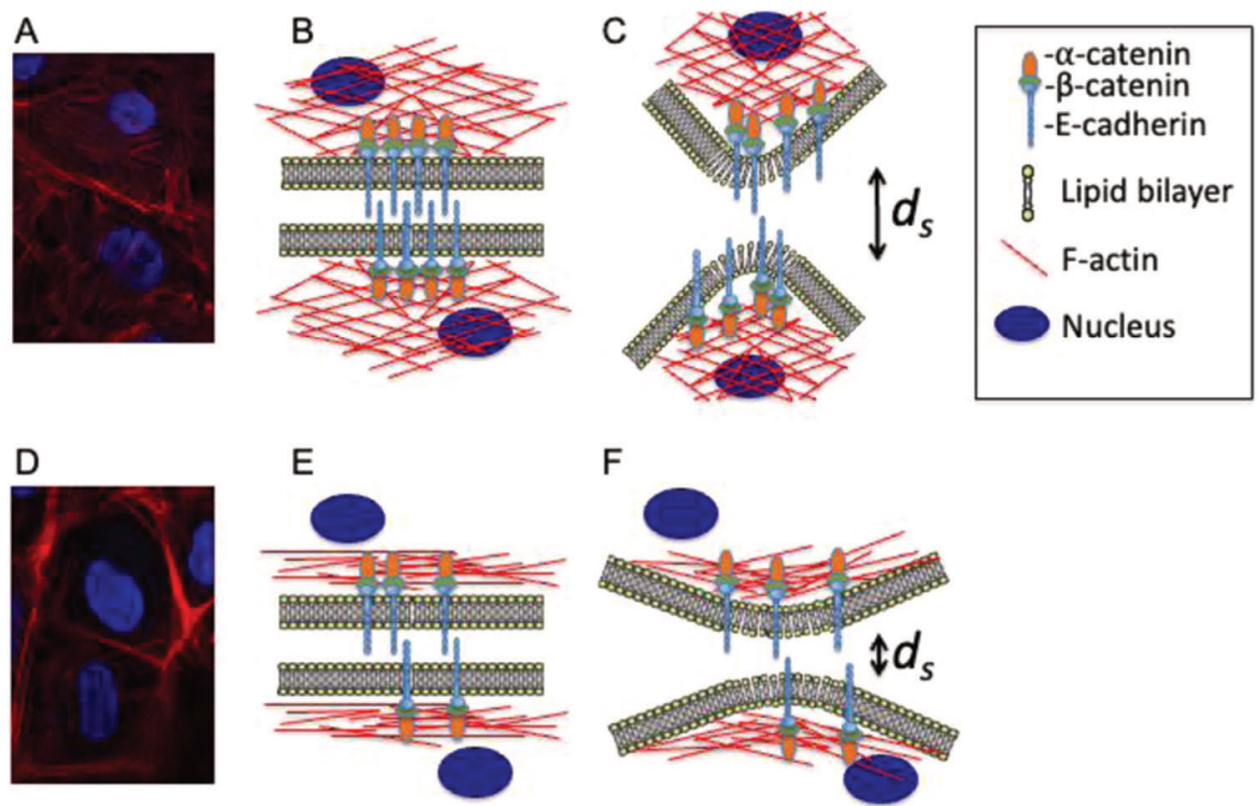

FIGURE 1. Schematic of E-cadherin based protein changes and cytoskeletal reorganization in healthy (A, B and C) and diseased (D, E and F) epithelial cells. In panels A and D, TRITC-conjugated phalloidin (red) is used to resolve gross cytoskeletal architecture whilst DAPI identifies nuclear staining (blue). In healthy cells the cytoskeleton appears as a transcellular filamentous network (A), whilst diseased cells exhibit rigid peripheral stress fibers (D). In Phase 1 (B and E) cells are in contact, and ligation is formed. NB: E-cadherin expression is reduced in diseased cells (E). In Phase 2 (C and F) the separation process is shown, in which the interplay between cytomechanics and surface molecular binding results in a shorter distance of separation $\left(d_{s}\right)$, i.e., healthy cells are more elastic and demonstrate greater cell-cell tethering. 


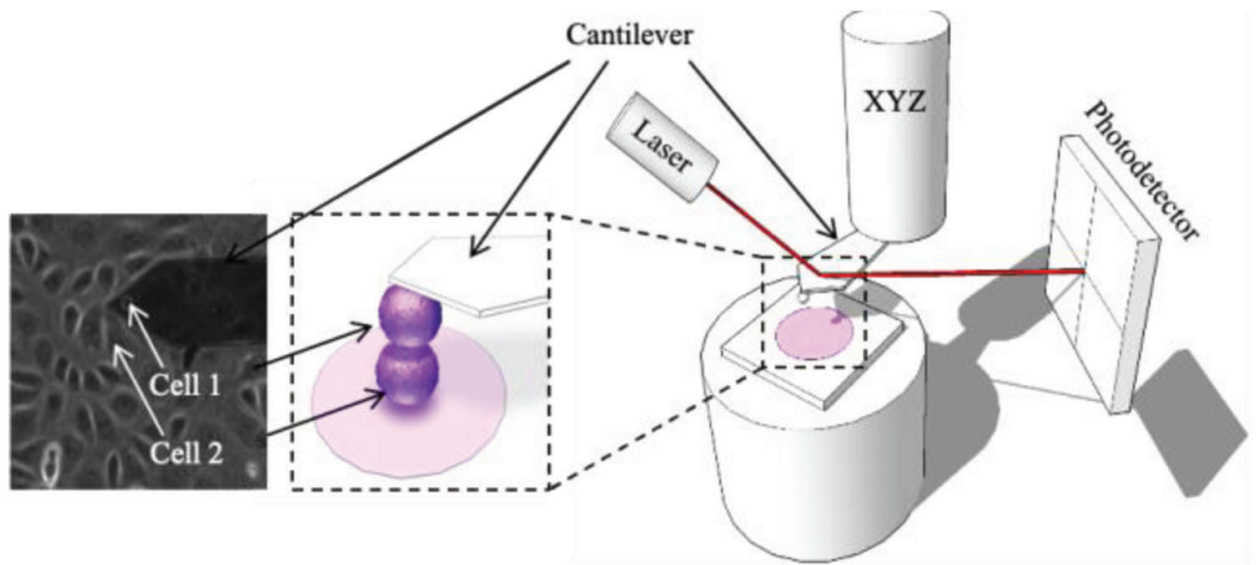

FIGURE 2. AFM single-cell force spectroscopy: A long-range piezoelectric actuator (XYZ) controls the position of the cantilever-based cell (Cell 1) while any resistance deflection is detected by movement of the laser on a photodetector. The cantilever-attached cell (Cell 1) is positioned over a substrate-attached cell (Cell 2). The cells are held together under a force of $1 \mathrm{nN}$ for $10 \mathrm{~s}$ to permit ligation of surface molecules before retracting the cantilever at a constant $10 \mu \mathrm{m} / \mathrm{s}$. This arrangement allows high-resolution continuous measurements of force $v$ s. displacement prior to complete cell-cell separation up to $100 \mu \mathrm{m}$.

considering the impact to physiological parameters. Substrate stiffness has been shown to play a major role in cell mechanosensitivity and cell phenotype (Iskratsch et al., 2014), and the response of cells to changes in matrix rigidity is regulated by local sensing at focal adhesion complexes and active and passive cytoskeletal stress (Doss et al., 2020). Understanding how culture conditions and substrate composition impact on cell behavior is a vital consideration when determining physiological responsiveness.

\section{Measuring Cell-Cell and Cell-Substrate Adhesion}

Atomic force microscopy (AFM) single cell force spectroscopy (SCFS) can be used to quantify mechanics and adhesion forces at the single-cell level and can provide accurate measurements of cellular deformation behaviour or compliance for many cell types, including renal tubular epithelial cells (Siamantouras et al., 2016). Changes in the resistance of a healthy or diseased cell to physical separation from its neighbor or designated substrate can be accurately measured (AFM-SCFS shown in Fig. 2; full protocol in Siamantouras et al. (2020). The system provides sufficient force-displacement ranges (up to $100 \mu \mathrm{m}$ ) to ensure precise detection of maximum unbinding forces of ligand-receptor interactions in cell-to-cell adhesion measurement, and configuration of the system for microbead indentation can be used to assess force-displacement curves to determine cell rigidity (Siamantouras et al., 2014).

\section{Conclusion}

Loss of cell-cell adhesion parameters is a function of both the energy associated with mechanical changes in cytoskeletal reorganisation and viscoelastic cell deformation, as well as the force required to unbind molecular surface contacts between cells and between cells and the ECM. Accurate determination of cell adhesion in disease presentation and/or progression requires more than simple estimates for changes in protein expression and should consider both biochemical and biophysical attributes of cell-cell and cellmatrix contacts.
Acknowledgement: $\mathrm{CEH}$ and PES thank the Renal Team within the Diabetes, Metabolism \& Inflammation Group, especially JA Potter, GW Price, CL Cliff and BM Williams who contributed background knowledge/assistance in support of the AFM studies described in this article.

Authors' Contribution: The authors confirm contribution to the paper as follows: study conception and design: PES and $\mathrm{CEH}$; data collection PES, $\mathrm{CEH}$ and ES; analysis and interpretation of results $\mathrm{PES}, \mathrm{CEH}$ and ES, draft manuscript preparation: PES and $\mathrm{CEH}$. All authors approved the final version of the manuscript.

Funding Statement: This work was supported by Diabetes UK who provided the AFM-SCFS system [12/0004546] and funded studies examining cell-cell [16/0005427, 18/0005919], and cell-ECM interactions [16/0005544].

Conflicts of Interest: The authors have no conflicts of interest to report regarding the present study.

\section{References}

Buckley CD, Tan J, Anderson KL, Hanein D, Volkmann N, Weis WI, Nelson WJ, Dunn AR (2014). The minimal cadherin-catenin complex binds to actin filaments under force. Science 346: 1254211. DOI 10.1126/science.1254211.

Cotrina ML, Lin JHC, Nedergaard M (2008). Adhesive properties of connexin hemichannels. Glia 56: 1791-1798. DOI 10.1002/ glia.20728.

Doss BL, Pan M, Gupta M, Grenci G, Mège RM, Lim CT, Sheetz MP, Voituriez R, Ladoux B (2020). Cell response to substrate rigidity is regulated by active and passive cytoskeletal stress. Proceedings of the National Academy of Sciences 117: 12817-12825. DOI 10.1073/pnas.1917555117.

Gkretsi V, Stylianopoulos T (2018). Cell adhesion and matrix stiffness: Coordinating cancer cell invasion and metastasis. Frontiers in Oncology 8: 145. DOI 10.3389/fonc.2018.00145.

Goodwin K, Lostchucka EE, Cramba KML, Zulueta-Coarasa T, Fernandez-Gonzalez R, Tanentzapf G (2017). Cell-cell and cell-extracellular matrix adhesions cooperate to organize actomyosin networks and maintain force transmission 
during dorsal closure. Molecular Biology of the Cell 28: 13011310. DOI 10.1091/mbc.e17-01-0033.

Hills CE, Squires PE (2011). The role of TGFB and epithelial-tomesenchymal transition in diabetic nephropathy. Cytokine \& Growth Factors Reviews 22: 131-139.

Hills CE, Siamantours E, Smith SW, Cockwell P, Liu KK, Squires PE (2012). TGF-beta modulates cell-cell communication in early epithelial-to-mesenchymal transition. Diabetologia 55: 812824. DOI 10.1007/s00125-011-2409-9.

Hills CE, Price GW, Wall MJ, Kauffman TJ, Tang SC, Yiu WH, Squires PE (2018). Transforming Growth Factor Beta 1 drives a switch in connexin mediated cell-to-cell communication in the diabetic kidney. Cellular Physiology and Biochemistry 45: 2369-2388. DOI 10.1159/000488185.

Iskratsch T, Wolfenson H, Sheetz MP (2014). Appreciating force and shape-The rise of mechanotransduction in cell biology. Nature Reviews Molecular Cell Biology 15: 825-833. DOI 10.1038/nrm3903.

Jaitovich A, Jaim Etcheverry G (2004). Adhesion molecules. Their role in cardiovascular physiopathology. Medicina 64: 455-462.

Kassianidou E, Kumar S (2015). A biomechanical perspective on stress fiber structure and function. Biochimica Biophysica Acta 1853: 3065-3074. DOI 10.1016/j.bbamcr.2015.04.006.

Kechagia JZ, Ivaska J, Roca-Cusachs P (2019). Integrins as biomechanical sensors of the microenvironment. Nature Reviews Molecular Cell Biology 20: 457-473. DOI 10.1038/ s41580-019-0134-2.

Lin JHC, Takano T, Cotrina ML, Arcuino G, Kang J et al. (2002). Connexin 43 enhances the adhesivity and mediates the invasion of malignant. Glioma cells. Journal of Neuroscience 22: 4302-4311.

Potter JA, Price GW, Cliff CL, Green CR, Squires PE, Hills CE (2021). Collagen I drives ECM remodeling via $\mathrm{Cx} 43$ hemichannel ATP release. International Journal of Molecular Sciences 22: 3644. DOI 10.3390/ijms22073644.

Price GW, Chadjichristos CE, Kavvadas P, Tang SW, Yiu WH et al. (2020). Blocking Connexin-43 mediated hemichannel activity protects against early tubular injury in experimental chronic kidney disease. Cell Communication \& Signaling 18: 79. DOI 10.1186/s12964-020-00558-1.

Scarpa E, Szabó A, Bibonne A, Theveneau E, Parsons M, Mayor R (2015). Cadherin switch during EMT in neural crest cells leads to contact inhibition of locomotion via repolarization of forces. Developmental Cell 34: 421-434. DOI 10.1016/j.devcel.2015.06.012.

Siamantouras E, Hills CE, Younis MYG, Squires PE, Liu KK (2014). Quantitative investigation of calcimimetic R568 on beta-cell adhesion and mechanics using AFM single-cell force spectroscopy. FEBS Letters 588: 1178-1183. DOI 10.1016/j. febslet.2014.02.058.

Siamantouras E, Hills CE, Squires PE, Liu KK (2015). Nanomechanical investigation of soft biological cell adhesion using atomic force microscopy. Cellular \& Molecular Bioengineering 8: 22-31. DOI 10.1007/s12195-014-0359-2.

Siamantouras E, Hills CE, Squires PE, Liu KK (2016). Quantifying cellular mechanics and adhesion in renal tubular injury using single cell force spectroscopy. Nanomedicine: Nanotechnology, Biology and Medicine 12: 1013-1021. DOI 10.1016/j.nano.2015.12.362.

Siamantouras E, Price GW, Potter JA, Hills CE, Squires PE (2019). Purinergic receptor (P2X7) activation reduces cell-cell adhesion between tubular epithelial cells of the proximal kidney. Nanomedicine: Nanotechnology, Biology, and Medicine 22: 102108. DOI 10.1016/j.nano.2019.102108.

Siamantouras E, Hills CE, Liu KK, Squires PE (2020). Examining cell-cell interactions in the kidney using AFM single-cell force spectroscopy. In: Gnudi L, Long D (eds.), Diabetic nephropathy. methods in molecular biology 2067. New York, NY: Humana.

Strauss RE, Gourdie RG (2020). Cx43 and the actin cytoskeleton: novel roles and implications for cell-cell junction-based barrier function regulation. Biomolecules 10: 1656. DOI 10.3390/biom10121656.

Theocharis AD, Manou D, Karamanos NK (2019). The extracellular matrix as a multitasking player in disease. FEBS Journal 286: 2830-2869. DOI 10.1111/febs.14818.

Wennstrom M, Nielsen HM (2012). Cell adhesion mole-cules in Alzheimer's disease. Degenerative Neurological Neuromuscular Disease 2: 65-77. 